\title{
Tractable Transport Calculations through Artificial Dissipation
}

\section{Dissipation-assisted operator evolution method for capturing hydrodynamic transport}

Authors: Tibor Rakovszky, C. W. von Keyserlingk, and Frank Pollmann

Phys. Rev. B 105, 075131 (2022)

\section{Operator backflow and the classical simulation of quantum transport} Authors: C. W. von Keyserlingk, Frank Pollmann, and Tibor Rakovszky arXiv:2111.09904

\section{Recommended with a Commentary by E. Miles Stoudenmire, Center for Computational Quantum Physics, Flatiron Institute, 162 5th Avenue, New York, NY 10010, USA}

The dynamics of thermalizing, out-of-equilibrium quantum systems harbors a wealth of open questions about transport properties, operator scrambling, spreading of entanglement, and complexity of classical simulation [1,2]. While significant progress has been made in special cases such as integrable systems [3], to understand more generic systems numerical calculations play a major role. However, there are major limitations when considering the existing landscape of numerical techniques.

A large body of numerical work on thermalization and long-time dynamics of many-body systems has relied on exactly diagonalizing the Hamiltonian to obtain the full spectrum $[4,5,6]$, allowing one to reach arbitrary times and temperatures but severely limiting the system sizes that can be addressed.

A promising alternative is using matrix product state (MPS) techniques $[7,8]$ to study the dynamics of one-dimensional systems. MPS are a factorized representation of a quantum state into a chain of matrix products, with different matrices placed into the chain depending on the amplitude one wishes to compute. An MPS is also one of the simplest examples of a tensor network. In one dimension, MPS scale to large or even infinite system sizes, and there are a variety of controlled algorithms to evolve them to moderate times and to include finite temperature effects $[9,10,11,12]$. But simulating out-of-equilibrium dynamics with MPS to long times encounters a serious problem: under unitary, closed-system evolution, the entanglement of most quantum states grows quickly and eventually becomes proportional to the number of sites of the system [13]. * Because MPS are a compression technique relying

${ }^{*}$ This is in sharp contrast to one-dimensional ground states where the entanglement is independent of system size. 
on low entanglement, such a rapid growth is a disaster and leads to an exponential blow up of both memory and computational costs.

However, there have been a number of clues that MPS time evolution may be "fixable" for reaching the long-time, hydrodynamic regime of thermalizing systems. One clue was the development of an intriguing algorithm for time-evolving MPS which projects the full Hilbert space dynamics into the manifold of MPS having a fixed number of parameters. This algorithm, known as the time-dependent variational principle (TDVP) [12], realizes a kind of semiclassical dynamics within the space of MPS states which exactly conserves energy and exhibits chaotic behavior, while having a precise connection back to the true quantum dynamics. Numerically integrating the TDVP equations was conjectured to be sufficient to capture transport coefficients, such as the energy diffusion constant, even when working at a fixed level of approximation [14]. However, follow up work showed that such a fixed-approximation approach can fail to capture the true diffusion constant and a more systematic extrapolation is needed in general [15].

Another important clue that MPS techniques might be extended to capture long-time dynamics was the realization that thermal mixed states can generically be captured by an MPS if one works within a doubled Hilbert space - a so-called purification [16]. (Purifying the system is equivalent to working with an operator extension of MPS, called a matrix product operator or MPO.) Since non-integrable systems generically thermalize at long times, one might hope that evolution of a pure state represented as an MPS can be smoothly connected to a mixed-state description at longer times without encountering a high-entanglement regime of the time evolution that cannot be numerically handled. Or that by working with mixed states or in a Heisenberg picture of evolving operators from the beginning one can handle the computation at all times.

Indeed such operator-centric MPS (or MPO) approaches to efficiently studying long-time dynamics have begun to appear $[17,18,19,20,21,22]$. One important realization was that the usual compression or truncation developed for MPS when applied to mixed states is not sufficient for controlling the growth of entanglement, even though the long-time mixed state should fit within the space of MPS. One way to understand this point is that unitary dynamics is reversible, while a thermal Gibbs states has no "memory" of the particular initial state it evolved from. Thus some of the newly proposed approaches [17, 18, 19, 20, 21, 22] are distinguished by their relatively aggressive truncation procedures that dispense with reversability. For example, the density matrix truncation algorithm proposed by White et al. [18] discards a small piece of the mixed state after each time step, although it crucially preserves local, overlapping reduced density matrices as well as the overall unit trace.

Rakovszky, Keyserlingk, and Pollmann [22] have proposed a different operator-based evolution procedure they call dissipation assisted operator evolution (DAOE) that is compelling for its simplicity: within a Heisenberg picture of evolving the operators whose dynamics one wants to measure, the dynamics is expressed within the basis of strings of Pauli matrices. As a choice of basis, it is straightforward to implement with MPS techniques. But it gives a clever advantage: one can implement an artificial dissipation step in a numerically efficient way. The details are technical, but one can exactly define another, efficiently implementable tensor network which acts on the operator being evolved so as to suppress all operator strings whose length is greater than some length $\ell^{*}$. More precisely, if one writes the operator being evolved at a certain time as $O(t)=\sum c_{S}(t) S$ where each $S$ is a string of Pauli operators 
and $\ell_{S}$ its length ${ }^{\dagger}$, then after being acted by the dissipation superoperator $\mathcal{D}_{\ell^{*}, \gamma}$ the new operator $O^{\prime}(t)$ after dissipation becomes

$$
O^{\prime}(t)=\mathcal{D}_{\ell^{*}, \gamma}[O(t)]=\sum_{S, \ell_{S} \leq \ell^{*}} c_{S}(t) S+\sum_{S, \ell_{S}>\ell^{*}} e^{-\gamma\left(\ell_{S}-\ell^{*}\right)} c_{S}(t) S
$$

Operator strings whose lengths are less than $\ell^{*}$ are unaffected, while longer operator strings are exponentially suppressed. The dissipation step is applied periodically during the timeevolution procedure, so that the actual amount of dissipation depends on the ratio of the period of dissipationless evolution to the dissipation strength $\gamma$.

Though the dynamics are now artificially deformed from the true unitary dynamics, one can hope to extrapolate in the dissipation strength $\gamma$, working at different values of $\ell^{*}$. Most importantly, the whole point of introducing the dissipation is that one hopes it will make the dynamics more numerically tractable by reducing the complexity of the state the MPS must capture. This hope is actually realized: during the evolution, the entanglement of the MPS used to represent the operator first increases, but then saturates to a fixed longtime value, allowing the dissipative dynamics to be run at fixed accuracy for arbitrarily long times and for large system sizes. Yet despite the artificial nature of the dynamics, for small enough dissipation strengths $\gamma$, Rakovszky et al. confirm that key hydrodynamical properties, namely the spreading of correlation functions, agree very well at short times with exact results on smaller systems. For model one-dimensional spin chain systems and in the infinite temperature ensemble, the authors use their approach with an extrapolation in $\gamma$ to estimate diffusion constants describing long-time transport of conserved quantities (energy or magnetization), finding good agreement with previous estimates.

In a recent follow-up [23], these authors now give a more detailed justification for their dissipation-assisted operator evolution procedure, focusing on "backflow corrections" meaning the process of longer operator strings yielding corrections to the coefficients of shorter operator strings later on in time. The main conclusion is that such corrections are suppressed exponentially in the cutoff length $\ell^{*}$, justifying it as a parameter that can be used to make reliable extrapolations in generic settings. A rough explanation of the argument is that the long-time behavior of correlation functions can be expanded in terms of the dynamics of the most slowly-evolving operators with which they have non-zero overlap. These slow operators are related to the local conserved quantities of the system such as magnetization or charge. In contrast, time-dependent processes which involve longer strings of Pauli operators of length $\ell$ are exponentially suppressed in $\ell$, and only those with $\ell>\ell^{*}$ are affected by the artificial dissipation process of DAOE, making its cumulative effect exponentially small too. The authors support these claims with both theoretical arguments based on hydrodynamics and with numerical experiments on a spin chain and a symmetric random unitary circuit model.

Looking into the future, Keyserlignk, Pollmann, and Rakovszky ask if the success of their approach could indicate that hydrodynamic transport properties of ergodic systems are efficiently classically simulable under general assumptions [23]. However, more work is needed to show their approach works well for finite-temperature systems and in wider classes of

\footnotetext{
${ }^{\dagger}$ The length of a Pauli operator string is the number of non-identity Pauli operators occuring within it, regardless of spatial location.
} 
models. Also, it would be interesting to see if other related approximation strategies such as that of White et al. [18] can be connected to the DAOE approach, to give a more comprehensive algorithmic view of how thermalization and long-time hydrodynamical behavior emerge within a concrete MPS or tensor network framework.

\section{References}

[1] Luca D'Alessio, Yariv Kafri, Anatoli Polkovnikov, and Marcos Rigol. From quantum chaos and eigenstate thermalization to statistical mechanics and thermodynamics. Advances in Physics, 65(3):239-362, 2016.

[2] B. Bertini, F. Heidrich-Meisner, C. Karrasch, T. Prosen, R. Steinigeweg, and M. Žnidarič. Finite-temperature transport in one-dimensional quantum lattice models. Rev. Mod. Phys., 93:025003, May 2021.

[3] Romain Vasseur and Joel E Moore. Nonequilibrium quantum dynamics and transport: from integrability to many-body localization. Journal of Statistical Mechanics: Theory and Experiment, 2016(6):064010, jun 2016.

[4] Marcos Rigol, Vanja Dunjko, and Maxim Olshanii. Thermalization and its mechanism for generic isolated quantum systems. Nature, 452(7189):854-858, 2008.

[5] Jens H. Bardarson, Frank Pollmann, and Joel E. Moore. Unbounded growth of entanglement in models of many-body localization. Phys. Rev. Lett., 109:017202, Jul 2012.

[6] Yevgeny Bar Lev, Guy Cohen, and David R. Reichman. Absence of diffusion in an interacting system of spinless fermions on a one-dimensional disordered lattice. Phys. Rev. Lett., 114:100601, Mar 2015.

[7] Román Orús. A practical introduction to tensor networks: Matrix product states and projected entangled pair states. Annals of physics, 349:117-158, 2014.

[8] Ulrich Schollwöck. The density-matrix renormalization group in the age of matrix product states. Annals of Physics, 326(1):96-192, 2011. January 2011 Special Issue.

[9] Steven R. White and Adrian E. Feiguin. Real-time evolution using the density matrix renormalization group. Phys. Rev. Lett., 93:076401, Aug 2004.

[10] Andrew John Daley, Corinna Kollath, Ulrich Schollwöck, and Guifré Vidal. Timedependent density-matrix renormalization-group using adaptive effective hilbert spaces. Journal of Statistical Mechanics: Theory and Experiment, 2004(04):P04005, 2004.

[11] Adrian E. Feiguin and Steven R. White. Finite-temperature density matrix renormalization using an enlarged hilbert space. Phys. Rev. B, 72:220401, Dec 2005.

[12] Jutho Haegeman, J. Ignacio Cirac, Tobias J. Osborne, Iztok Pizorn, Henri Verschelde, and Frank Verstraete. Time-dependent variational principle for quantum lattices. Phys. Rev. Lett., 107:070601, Aug 2011. 
[13] T. Prosen and M. Znidaric. Is the efficiency of classical simulations of quantum dynamics related to integrability? Phys. Rev. E, 75:015202, Jan 2007.

[14] Eyal Leviatan, Frank Pollmann, Jens H. Bardarson, David A. Huse, and Ehud Altman. Quantum thermalization dynamics with matrix-product states, 2017. arxiv:1702.08894.

[15] Benedikt Kloss, Yevgeny Bar Lev, and David Reichman. Time-dependent variational principle in matrix-product state manifolds: Pitfalls and potential. Phys. Rev. B, 97:024307, Jan 2018.

[16] M. Znidaric, T. Prosen, and I. Pizorn. Complexity of thermal states in quantum spin chains. Phys. Rev. A, 78:022103, Aug 2008.

[17] T Prosen and M Znidaric. Matrix product simulations of non-equilibrium steady states of quantum spin chains. Journal of Statistical Mechanics: Theory and Experiment, 2009(02):P02035, feb 2009.

[18] Christopher David White, Michael Zaletel, Roger S. K. Mong, and Gil Refael. Quantum dynamics of thermalizing systems. Phys. Rev. B, 97:035127, Jan 2018.

[19] Daniel E. Parker, Xiangyu Cao, Alexander Avdoshkin, Thomas Scaffidi, and Ehud Altman. A universal operator growth hypothesis. Phys. Rev. X, 9:041017, Oct 2019.

[20] Thomas Klein Kvorning, Loïc Herviou, and Jens H. Bardarson. Time-evolution of local information: thermalization dynamics of local observables, 2021. arxiv:2105.11206.

[21] Christopher David White. Effective dissipation rate in a liouvillean graph picture of high-temperature quantum hydrodynamics, 2021. arxiv:2108.00019.

[22] Tibor Rakovszky, C. W. von Keyserlingk, and Frank Pollmann. Dissipation-assisted operator evolution method for capturing hydrodynamic transport. Phys. Rev. B, 105:075131, Feb 2022.

[23] C. W. von Keyserlingk, Frank Pollmann, and Tibor Rakovszky. Operator backflow and the classical simulation of quantum transport, 2021. arxiv:2111.09904. 